\section{e-Journal of Educational}

Research, Assessment and Evaluation

\section{REIIEVE}

Revista ELectrónica de Investigación y EValuación Educativa

\title{
ADAPTACIÓN AL ESPACIO EUROPEO DE EDUCACIÓN SUPERIOR DE UN CUESTIONARIO DE OPINIÓN DEL ALUMNADO SOBRE LA DOCENCIA DE SU PROFESORADO
}

\section{[Adapting to the European Higher Education Area a questionnaire on student opinion about the teaching of lecturers]}

by/por

$\underline{\text { Article record }}$

About authors

$\underline{\text { HTML format }}$

\author{
Lukas, José Francisco (if.lukas@ehu.es) \\ Santiago, Karlos (karlos.santiago@ehu.es) \\ Etxeberria, Juan (juanito@ehu.es) \\ Lizasoain, Luis (luis.lizasoain@ehu.es)
}

\section{$\underline{\text { Ficha del artículo }}$ \\ Sobre los autores \\ Formato HTML}

\begin{abstract}
The aim of this work is to present the adaptation to the European Higher Education Area of a questionnaire on the opinions of University of the Basque Country students about their lecturers teaching process. This process undertaken at the University used a mixed methodology approach. After reviewing questionnaires from other universities, taking into account the theoretical framework of the new questionnaire, the transversal skills adopted by the University and the three dimensions set out by ANECA, a series of indicators and possible items deemed suitable to be included in the new questionnaire were drawn up. This theoretical framework, the indicators and the items were all compared and contrasted within various focus groups undertaken with experts in the topic, with lecturers from the different areas of the University, and with students. Based on this comparison, the pilot questionnaire, which had 18 items plus two more criterion-referenced items, was drawn up. This was applied to a sample of almost one thousand students. The analyses carried out to compare the efficacy of the items, as well as the reliability and the validity of the test, show that the questionnaire rigorously complies with the standards required by this type of instrument. Finally, in the discussion of the results, certain controversial aspects, or those relating to improving the evaluation of the university teaching staff, are presented. Indicated amongst these aspects, is the need to incorporate the questionnaire into a more wider-ranging evaluation plan, such as DOCENTIA, the possibility of creating
\end{abstract}

\section{Resumen}

El objetivo de este trabajo es presentar el proceso de adaptación al Espacio Europeo de Educación Superior del cuestionario de opinión del alumnado sobre la docencia de su profesorado que se ha seguido en la Universidad del País Vasco. En este proceso se ha adoptado una metodología mixta. Tras efectuar la revisión de los cuestionarios de otras universidades, teniendo en cuenta el marco teórico del nuevo cuestionario, las competencias transversales adoptadas por la Universidad y las tres dimensiones que señala la ANECA, se redactaron una serie de indicadores y posibles ítems susceptibles de conformar el nuevo cuestionario. Este marco teórico, los indicadores e ítems fueron contrastados en diversos grupos de discusión realizados con expertos en el tema, profesorado de distintas áreas de la propia universidad y alumnado. A partir de este contraste se construyó el cuestionario piloto. El mismo consta de 18 ítems más dos ítems criterio. Fue aplicado a una muestra cercana al millar de alumnos. Los análisis realizados para comprobar la eficacia de los ítems, así como la fiabilidad y la validez de la prueba señalan que el cuestionario cumple con rigurosidad los estándares exigidos a este tipo de instrumentos. Por último, en la discusión de los resultados, se presentan algunos aspectos de controversia o mejora de la evaluación del profesorado universitario. Entre otros, se señala la necesidad de insertar el cuestionario en un plan de evaluación más amplio como DOCENTIA, la posibilidad de crear bancos de ítems, la inclusión de ítems abiertos o la necesidad de realizar apli- 
banks of items, the inclusion of open items and the exigency of undertaking on-line applications of the questionnaire.

\section{Keywords}

Teacher evaluation; European Higher Education Area; survey validation; reliability; validity; mixed methods. caciones on-line del cuestionario.

\section{Descriptores}

Evaluación del profesorado, Espacio Europeo de Educación Superior, validación de encuesta, fiabilidad, validez, métodos mixtos.
El llamado Espacio Europeo de Educación Superior (EEES) responde a un intento de armonizar los distintos sistemas universitarios de la Unión Europea creando una única medida del trabajo académico (el ECTS, European Credit Transfer System), facilitando el intercambio de estudiantes y el reconocimiento mutuo de titulaciones. Se inició en 1999 con el acuerdo de Bolonia. La adaptación que han hecho las universidades a este espacio ha acarreado una nueva visión y unas nuevas formas de enseñar y aprender (De la Fuente, Martínez, Peralta y García, 2010).

Se ha pasado de una enseñanza centrada en el profesor a proponer metodologías más activas en las que el estudiante es el actor de su propio aprendizaje. Todo ello ha supuesto que la función del docente haya cambiado. Del profesor transmisor de conocimientos se ha pasado al profesor orientador que guía el aprendizaje significativo de los estudiantes. Del profesor que evalúa (únicamente de manera sumativa) al final del proceso, normalmente mediante un examen, se ha pasado al docente que recoge información del proceso de aprendizaje del estudiante y le proporciona información para mejorar (evaluación formativa). Del profesor centrado únicamente en la enseñanza de los contenidos de su materia se ha pasado al docente que ha de tratar de guiar el desarrollo de ciertas competencias en sus estudiantes. Competencias que están en algunos casos íntimamente relacionadas con su materia, pero también competencias transversales adoptadas por la titulación o la propia universidad que han de desarrollarse. Es decir, del énfasis en la información de la materia específica se ha pasado a un enfoque más centrado en la forma- ción general del estudiante (Palazón, 2011) en donde las competencias transversales tienen un espacio relevante. Entre estas competencias, podemos señalar como ejemplos, desarrollar una actitud crítica, fomentar la expresión oral $\mathrm{y} / \mathrm{o}$ escrita, trabajar en equipo, etc.

Con objeto de mejorar la calidad de la enseñanza universitaria, la evaluación de la docencia universitaria es un componente muy importante (Molero y Ruiz, 2005). Tejedor (2003) plantea que cualquier propuesta de evaluación del profesorado debería tener en cuenta, entre otros, los aspectos siguientes: la actividad instructiva, la actividad investigadora, las condiciones de trabajo del docente y que informe al docente para ayudarle a mejorar.

Aunque para evaluar la docencia universitaria, hay diferentes estrategias tales como el rendimiento de los estudiantes, la autoevaluación, las técnicas de encuesta, el portafolio, la evaluación por expertos, etc., desde hace varias décadas en las universidades españolas se evalúa la docencia del profesorado mediante las encuestas de opinión que responden los estudiantes al final de cada proceso de enseñanza-aprendizaje. Hoy en día las valoraciones que hacen los estudiantes sobre la docencia de su profesorado son un indicador muy utilizado de calidad docente (Worthington, 2002; Tejedor, 2009; Gómez-Gallego, Gómez-Gallego, Pérez-Cárceles, Palazón-Pérez de los Cobos y Gómez-García, 2013). La calidad docente está ligada a la calidad de los docentes y de sus actuaciones (Lukas y Santiago, 2009). Sin embargo, estos cuestionarios no están, en la mayoría de los casos, adaptados a las nuevas metodologías formuladas desde el EEES. Obviamente, la propia universidad debe tener proce- 
dimientos y herramientas de contrastada calidad metodológica para evaluar a su profesorado (Palazón, 2011). En el presente artículo presentamos el proceso seguido en la Universidad del País Vasco (UPV/EHU) para adaptar el cuestionario a la nueva situación.

Previamente a la aplicación de la encuesta y de los análisis estadísticos correspondientes para validarla, surge la pregunta de qué es lo que debe incluir la misma. Es necesario indicar tal y como señala Tejedor (2009) que no existe un amplio consenso sobre lo que es un "buen profesor" y por lo tanto no está claro qué debe incluir la encuesta. Aunque resulte extraño no hay unanimidad cuando se trata de definir qué es una docencia de calidad (Ruiz Carrascosa, 2005).

Por otro lado, ¿qué aspectos de la calidad del docente son susceptibles de ser valorados por el estudiante? ¿Podría pensarse en una única cuestión para que la valore el estudiante del tipo "valora en una escala de 0 a 10 la actuación docente del profesor o profesora"? O, por el contrario puede pensarse que en la valoración del profesor o de la profesora existen diferentes dimensiones que han de ser abordadas.

Tal y como señalan Apodaca y Grad (2002), la polémica del carácter unidimensional o multidimensional de estas encuestas ha estado estrechamente relacionada con los resultados obtenidos mediante el análisis factorial exploratorio y confirmatorio, dando por sentado que este último se ha demostrado dudoso debido a que su flexibilidad permite aproximaciones sucesivas al modelo a contrastar. El asunto no es baladí puesto que está relacionado con el tipo de evaluación que se quiere plantear. Desde una perspectiva sumativa interesaría un cuestionario que ofreciera una única puntuación con la que poder valorar al docente (Berk, 2013). Sin embargo, desde un punto de vista formativo, si el propósito es mejorar las actuaciones docentes, parece que obtener valoraciones en diferentes dimensiones es más conveniente. En todo caso, como señalan esos mis- mos autores, ninguna técnica de análisis empírico puede sustituir la elaboración teórica previa del constructo de actuación docente.

La Agencia Nacional Española de Evaluación de la Calidad y Acreditación (ANECA) es una institución de carácter estatal cuyo objetivo es la mejora de la calidad del sistema de enseñanza superior y es la responsable de la evaluación, certificación y acreditación de docentes y titulaciones. En un documento sobre la evaluación de la actividad docente del profesorado universitario (ANECA, 2006), se señala que la misma debe ser concebida como parte de un sistema desarrollado por la propia institución que garantice la eficacia de sus planes de estudio. Por lo tanto tiene que estar inmersa en la política de profesorado y está íntimamente relacionada además de con la evaluación de la actividad docente, con la formación, innovación, promoción y/o mejoras salariales.

El Servicio de Evaluación Docente (SED) de la UPV/EHU se encarga, entre otras funciones, de gestionar el Cuestionario de Opinión al Alumnado sobre la Docencia del Profesorado, que surgió en el curso 1988/89 con un carácter voluntario y que, tras varias modificaciones, en los últimos años se ha ido aplicando en todos los centros de la universidad y está incorporado con total normalidad a la actividad cotidiana de la universidad.

En la UPV/EHU, desde el curso 2006/07, se ha aplicado un cuestionario de opinión al alumnado que ha respondido al paradigma educativo requerido por el EEES. Este hecho ha supuesto la antesala del diseño de este nuevo cuestionario que se pretende sea más ajustado al modelo adoptado por la propia universidad (denominado IKD; acrónimo de los términos en vasco para Aprendizaje Cooperativo y Dinámico), y que supone un proceso de enseñanza-aprendizaje cooperativo y dinámico centrado en el alumnado y que se configura como propuesta para el desarrollo curricular de las enseñanzas. Las características del modelo IKD son las siguientes: 
- Es dinámico y activo. Tiene su centro de gravedad en al aprendizaje del alumnado a través de metodologías activas y con el apoyo de las tecnologías de la información y comunicación, fomentando el aprendizaje en un contexto de enseñanza plurilingüe.

- Es plural y deberá interpretarse en cada titulación y en cada centro docente de forma flexible pero respondiendo a las señas de identidad de la universidad.

- Está basado en el fomento de la cooperación entre los diferentes agentes implicados en la docencia: alumnado, profesorado, personal de administración y servicios, departamentos, centros y agentes sociales.

- Para la construcción y desarrollo del modelo IKD existen diferentes herramientas entre las que cabe destacar: los programas formativos (AICRE: Asesoramiento para la Introducción del CRédito Europeo; SICRE: Seguimiento para la Introducción del CRédito Europeo; ERAGIN: Programa de formación del profesorado en metodologías activas de enseñanza; BEHATU: Programa de movilidad para la innovación educativa; FOPU: FOrmación docente del Profesorado Universitario), los proyectos de apoyo a la innovación educativa (PIE: Proyectos de Innovación Educativa) y las herramientas de evaluación de la docencia (DOCENTIA: Programa de apoyo a la evaluación de la actividad docente del profesorado universitario).

Este nuevo marco de enseñanza-aprendizaje conlleva, por lo tanto, una reorientación de las prácticas docentes del profesorado. Programas de evaluación de la actividad docente como DOCENTIA pretenden ser programas formativos para el profesorado, en tanto en cuanto guían a estos hacia aquellas prácticas y competencias que la institución considera "deseables".

El programa DOCENTIA, es una propuesta de la ANECA cuyo objeto es apoyar a las universidades en el diseño de mecanismos propios para gestionar la calidad de la actividad docen- te del profesorado universitario y favorecer su desarrollo y reconocimiento.

Dicho enfoque consideraría el modo en que el profesor planifica, desarrolla y mejora la enseñanza y lo que los estudiantes aprenden. El modelo en el que se fundamenta el programa DOCENTIA considera tres dimensiones como objeto de evaluación de la actividad docente: la planificación de la docencia, el desarrollo de la enseñanza y los resultados. Así, en DOCENTIA se valora que el profesorado planifique adecuadamente sus prácticas docentes, elaborando una Guía del estudiante que incluya la información necesaria para el seguimiento de la materia. Además, se intenta que los resultados de sus prácticas docentes se reflejen en la opinión de su alumnado y en las tasas de éxito para que, a su vez, den medida de lo que el alumnado ha aprendido. Por último, se espera que el profesorado sea capaz de detectar sus áreas de mejora y formarse en aquellas líneas identificadas como deficitarias.

Ante este planteamiento vuelve a surgir la misma pregunta realizada previamente: ¿Qué debe plasmar un cuestionario que recoge la opinión del alumnado sobre la docencia que recibe?

El modelo educativo IKD y el programa de evaluación formativa DOCENTIA nos aportan numerosas pistas sobre las funciones que, en el marco de la convergencia con Europa, debe desarrollar un profesor o profesora. Con la implantación de los nuevos grados y postgrados se ve la necesidad de construir un cuestionario que, en primer lugar, responda al modelo educativo IKD por el que apuesta la universidad, con su correspondiente vertiente formativa y en segundo, que cumpla con las condiciones de fiabilidad y validez que deben acompañar a todo cuestionario bien diseñado.

Resulta fundamental considerar que todas las dimensiones que componen el cuestionario deben converger en un único objetivo: especificar una serie de acciones docentes que logren que los y las estudiantes aprendan. 
En consecuencia, como resultado se espera que el alumnado opine sobre la capacidad del profesorado de fomentar en ellos y ellas sus capacidades para lograr un aprendizaje significativo, tanto en el orden profesional como en el personal.

\section{Método}

El objetivo principal del presente estudio es construir un cuestionario de opinión al alumnado sobre la docencia de su profesorado que esté adaptado al EEES y a DOCENTIA. También se pretende que el cuestionario tenga en consideración las competencias transversales. Además, se pretende reducir el número de ítems del cuestionario que se está utilizando hasta ahora (27 ítems). Obviamente, todo ello contando con el consenso de los diferentes estamentos involucrados en la evaluación (alumnado, profesorado, técnicos y directivos) y asegurando la fiabilidad y validez del nuevo cuestionario en aras a garantizar la calidad científica del instrumento. De la misma manera, mediante el proceso seguido se pretende obtener información relativa a la evaluación del profesorado para la mejora de la misma.

El método utilizado para la adaptación del Cuestionario de Opinión al Alumnado sobre la docencia del profesorado de la UPV/EHU ha sido un método mixto en el que se han aplicado técnicas cualitativas y cuantitativas en la recogida y análisis de los datos. Este diseño es un diseño exploratorio secuencial, en terminología utilizada por Creswell y Plano (2011). En estos diseños se utiliza un método cualitativo para recoger toda la información necesaria para la aplicación del método cuantitativo previsto. En este caso, se han constituido grupos de discusión con objeto de recoger las opiniones de diferentes agentes universitarios (expertos y técnicos en evaluación, profesorado y alumnado). A continuación se ha construido el cuestionario de opinión, tras cuya aplicación se han realizado los correspondientes análisis estadísticos.

La utilización de técnicas cuantitativas y cualitativas ha posibilitado un conocimiento más preciso, variado, sólido y completo del fenómeno analizado. En definitiva, ha dado mayor credibilidad y validez al estudio. Además, el proceso de adaptación ha sido un proceso dinámico, participativo e interactivo en el que, como se verá más adelante, los diversos agentes participantes han tenido la oportunidad de dar su opinión. Opinión que ha sido tenida en cuenta y que ha tenido repercusión en distintas fases del proceso.

En el proceso de construcción del nuevo cuestionario se siguieron distintas estrategias. Por un lado se hizo una revisión bibliográfica de las competencias que debe tener el profesorado en el EEES. Paralelamente a esta revisión bibliográfica se analizó el modelo educativo de la UPV/EHU (modelo IKD) para tratar de determinar el modelo de profesorado al que aspira la propia universidad de cara a que el nuevo cuestionario tenga un enfoque formativo con el objetivo de recoger, en la medida de lo posible, aquellas acciones que conducen al desarrollo de una buena práctica docente.

Por otro lado, se hizo un estudio comparativo entre cuestionarios de diferentes universidades del Estado (Alcalá, Autónoma de Madrid, Cádiz, Complutense, Córdoba, Deusto, Extremadura, Huelva, Illes Balears, Jaén, A Coruña, La Laguna, León, Málaga, Navarra, Politécnica de Cataluña, Pública de Navarra, Salamanca, Santiago de Compostela, Sevilla, Mondragón, Europea de Madrid, Valencia, Valladolid, Zaragoza). Obviamente, también se ha considerado el cuestionario que propone la ANECA.

Tras la revisión realizada se elaboró un documento marco para su discusión con técnicos y dirección del SED de la UPV/EHU. En este documento se distinguieron las tres dimensiones que, en principio había de tener el nuevo cuestionario. A saber, Planificación de la docencia, Desarrollo de la enseñanza y Resultados. Estas dimensiones están en consonancia con la propuesta de la ANECA y contemplados en el programa DOCENTIA. A su vez, se tuvieron en cuenta los tres momentos que pue- 
den distinguirse en el proceso de enseñanzaaprendizaje, es decir, la preparación del proceso por parte del profesor (diseño de programa, guía docente...), el desarrollo de la docencia, es decir el momento o momentos donde el profesor o profesora y el alumnado están juntos $\mathrm{y}$, por último, los resultados obtenidos como fruto del proceso de enseñanzaaprendizaje. Junto con la definición de las dimensiones, se señalaron también los indicadores del proceso de enseñanza-aprendizaje susceptibles de ser evaluados en cada una. Obviamente, solo se incluyeron aquellos que pueden ser directamente percibidos por el alumnado. Por último, se presentaban los ítems clasificados según la dimensión a la que pertenecían. Además se ofrecían alternativas de otros ítems que pudieran ser adecuados para la nueva propuesta. A partir de la discusión se consensuó el documento a presentar al grupo de expertos.

La función que ha desempeñado el grupo de expertos ha sido la recogida y puesta en común en un grupo de discusión de las opiniones referentes al cuestionario actual, tanto sobre sus puntos fuertes a mantener como los cambios que deberían producirse en el mismo. Igualmente se ha reflexionado acerca del modelo de cuestionario que se ha presentado y cuestiones relacionadas con la evaluación del profesorado. Esta reflexión crítica ha ayudado a identificar los ítems susceptibles de ser reformulados o directamente eliminados así como a la formulación de los nuevos ítems que compondrán el nuevo instrumento adaptado al IKD y al EEES. A partir de la reflexión y aportaciones realizadas posteriormente por miembros de este grupo de expertos se formuló un primer cuestionario que se puso a disposición de diferentes agentes educativos (alumnado y profesorado) para su consideración con objeto de perfilar el que ha sido el cuestionario piloto.

En total se realizaron siete grupos de discusión con profesorado y alumnado. En cuatro de ellos participaron profesores y profesoras de diferentes facultades que se agruparon en función de las áreas en las que ejercían su docen- cia. Los otros tres grupos estuvieron compuestos por alumnas y alumnos de diferentes campus de la UPV/EHU. A partir de estos grupos de discusión se ha construido el cuestionario que iba a ser utilizado en la aplicación piloto. En el Anexo 1 puede observarse el documento final consensuado en los grupos de discusión. Hay que decir que este documento fue reformulándose a lo largo de los distintos grupos de discusión ya que a partir de las reflexiones que se realizaban en los grupos, la información que se consideraba que podría ser interesante era incluida en los grupos de discusión posteriores. Por tanto, se puede decir que esta fase ha sido dinámica e interactiva.

Para la redacción final de los ítems, siguiendo las indicaciones de Muñiz (2005), se ha atendido a la representatividad, relevancia, diversidad, claridad, sencillez y comprensibilidad de los mismos.

Tras el análisis de la información recogida en los grupos de discusión se construyó el cuestionario para la aplicación piloto (Anexo 2). El cuestionario, además de las instrucciones y el apartado para el código de la "Situación Docente", se compone de dos partes fundamentales: en primer lugar, los datos para la contextualización del grupo de alumnos y alumnas y en segundo, el conjunto de ítems acerca de los cuales el alumnado tendrá que manifestar su opinión.

En cuanto a los ítems, el cuestionario se compone de 18 reactivos que responden a las tres dimensiones definidas para el cuestionario y a las competencias transversales, además de 2 ítems criterio, lo que hace un total de 20 elementos. El alumnado debe valorar cada uno de los ítems en una escala de 1 a 5 , en función del menor o mayor grado de acuerdo con los aspectos de la docencia de su profesorado expresados en los mismos.

Con objeto de examinar las características del mismo y de analizar su funcionamiento, se realizó una aplicación piloto a una muestra de estudiantes de la UPV/EHU de diversos grados y post-grados. 
Para el diseño de dicha muestra se consideraron los siguientes estratos:

- El campo científico: los 5 habituales

- El nivel: grado (curso $1^{\circ}$ y $3^{\circ}$ )

Así mismo se aplicó el cuestionario a 5 grupos de máster de Investigación y a otros 5 de máster profesional (uno por cada campo en cada caso). En la Tabla 1 se concretan los 34 grupos que conforman la muestra inicial. Esta concreción se efectuó teniendo en cuenta la distribución del alumnado en los distintos campos de conocimiento y niveles de grado y posgrado.

Tabla 1.- Grupos participantes en la aplicación piloto

\begin{tabular}{lccccc}
\hline & $\begin{array}{c}\text { Ciencias } \\
\text { Sociales } \mathbf{y} \\
\text { Jurídicas }\end{array}$ & $\begin{array}{c}\text { Ciencias } \\
\text { Experimentales }\end{array}$ & $\begin{array}{c}\text { Enseñanzas } \\
\text { Técnicas }\end{array}$ & $\begin{array}{c}\text { Ciencias de la } \\
\text { Salud }\end{array}$ & Humanidades \\
\hline Curso $\mathbf{1}^{\circ}$ Grado & 3 & 2 & 3 & 2 & 2 \\
Curso $3^{\circ}$ Grado & 3 & 2 & 3 & 2 & 2 \\
Máster profesional & 1 & 1 & 1 & 1 & 1 \\
Máster investigación & 1 & 1 & 1 & 1 & 1 \\
\hline
\end{tabular}

En esta aplicación se recogieron respuestas de 970 estudiantes que se distribuyen en la forma que aparece en la Tabla 2.

Tabla 2.- Estudiantes participantes en la aplicación piloto

\begin{tabular}{|c|c|c|c|c|c|c|}
\hline \multirow[b]{2}{*}{$\begin{array}{l}\text { TITULACIÓN: } \\
\text { GRADO O MÁSTER }\end{array}$} & \multicolumn{5}{|c|}{ CAMPO CIENTÍFICO } & \multirow[b]{2}{*}{ Total } \\
\hline & $\begin{array}{c}\text { Ciencias } \\
\text { Experimentales }\end{array}$ & $\begin{array}{l}\text { Enseñanzas } \\
\text { Técnicas }\end{array}$ & $\begin{array}{l}\text { Ciencias de } \\
\text { la Salud }\end{array}$ & $\begin{array}{c}\text { Ciencias } \\
\text { Sociales y } \\
\text { Jurídicas }\end{array}$ & Humanidades & \\
\hline Grado & 183 & 98 & 176 & 274 & 117 & 848 \\
\hline Máster & 6 & 76 & 14 & 17 & 9 & 122 \\
\hline Total & 189 & 174 & 190 & 291 & 126 & 970 \\
\hline
\end{tabular}

Una vez efectuada la recogida de datos, para comprobar la validez de la muestra se compararon las características de la misma con las de la población en las variables caracterizadoras incluidas en ambos cuestionarios. En la tabla 5 se presentan los diferentes porcentajes, apareciendo en primer lugar los de la muestra y a continuación y entre paréntesis, los poblacionales. Como puede observarse, las distribuciones son muy similares. Obviamente no se han comparado los relativos al curso y a la edad.

Para el estudio de las propiedades psicométricas y de las características del cuestionario, se han realizado los siguientes análisis: Descripción del colectivo analizado, Análisis de la Fiabilidad del Cuestionario, Estructura factorial del Cuestionario y Criterios de validez del Cuestionario.

\section{Resultados}

Una vez eliminados los casos de respuesta constante, la muestra empleada para analizar el cuestionario piloto estuvo formada por 938 estudiantes que, en función de los criterios de estratificación adoptados en el proceso de muestreo, se distribuyen de la manera que aparece en las siguientes tablas. 
Lukas, José Francisco; Santiago, Karlos; Etxeberria, Juan \& Lizasoain, Luis (2014). Adaptación al Es-pacio Europeo de Educación Superior de un cuestionario de opinión del alumnado sobre la docencia de su profesorado.

RELIEVE, 20 (1), art. 3. DOI: 10.7203/relieve.20.1.3812

Tabla 3.- Participantes en función del área de conocimiento

\begin{tabular}{lcc}
\hline Campos científicos & Frecuencia & Porcentaje \\
\hline Ciencias Experimentales & 187 & 19,9 \\
Enseñanza Técnicas & 169 & 18,0 \\
Ciencias de la Salud & 181 & 19,3 \\
Ciencias Sociales y Jurídicas & 282 & 30,1 \\
Humanidades & 119 & 12,7 \\
\multicolumn{1}{c}{ Total } & 938 & 100,0 \\
\hline
\end{tabular}

Tabla 4.- Participantes en función del nivel de estudios

\begin{tabular}{|c|c|c|}
\hline Nivel (grado o postgrado): & Frecuencia & Porcentaje \\
\hline Grado & 824 & 87,8 \\
\hline Máster & 114 & 12,2 \\
\hline Total & 938 & 100,0 \\
\hline
\end{tabular}

Tabla 5.- Características contextuales de los participantes (entre paréntesis datos de la población)

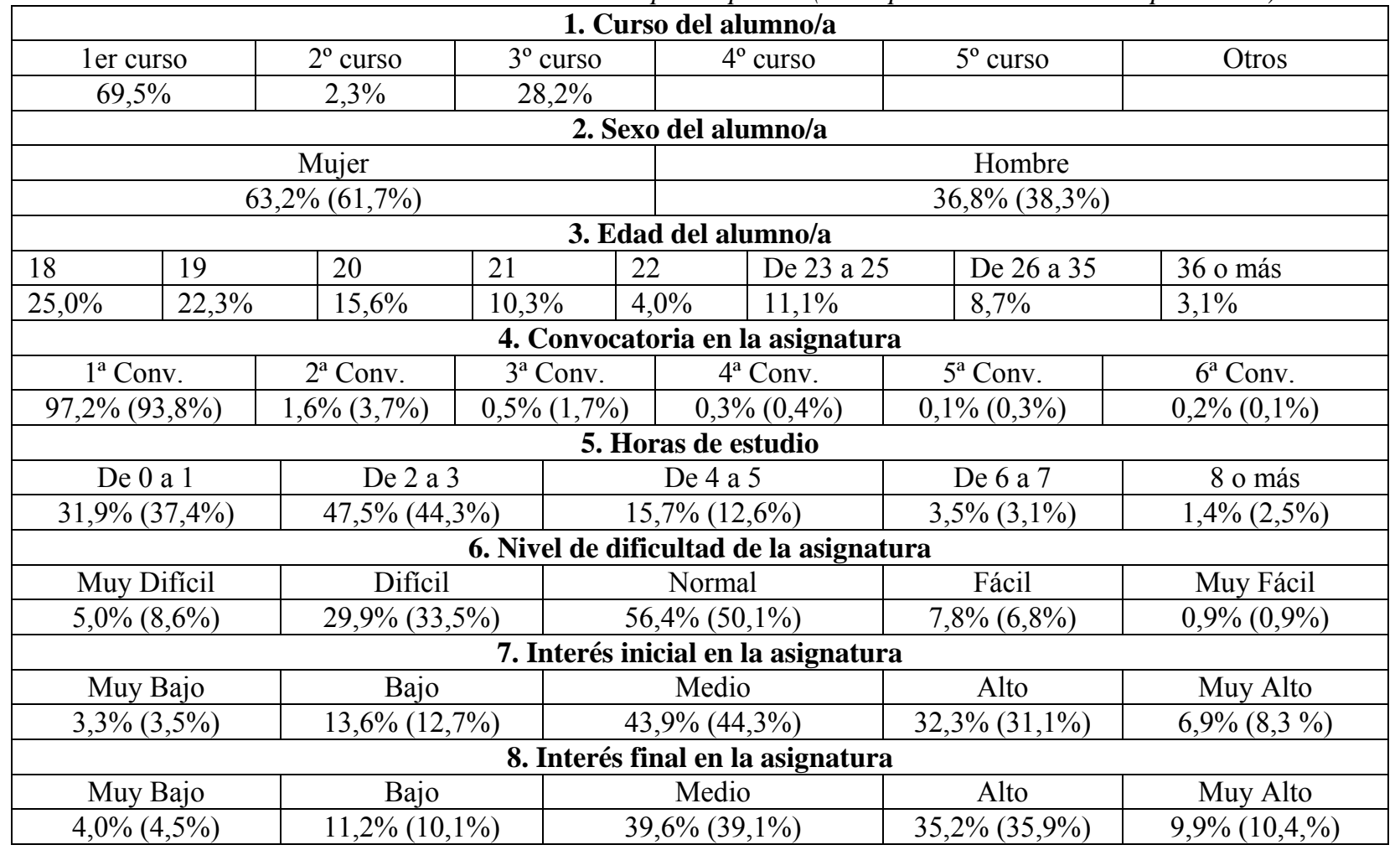

El cálculo del coeficiente de fiabilidad como consistencia interna del cuestionario se lleva a cabo mediante el estadístico Alfa de Cronbach y se ha calculado para el total $(\alpha=0,939)$ así como para cada segmento de la muestra objeto de estudio en función del campo científico. Como se puede observar en la Tabla 6 la fiabilidad del cuestionario es muy elevada y cons- tante lo que evidencia una alta consistencia de los elementos del instrumento. Aunque levemente inferiores en el campo de las enseñanzas técnicas, a la vista de la homogeneidad de los resultados en los diferentes campos científicos, efectuaremos el análisis de la contribución de cada ítem de forma conjunta obviando la diferenciación por ámbitos disciplinares. 
Tabla 6.- Coeficiente de fiabilidad del cuestionario en las diferentes áreas

\begin{tabular}{lcc}
\hline \multicolumn{1}{c}{ CAMPO CIENTÍFICO } & Alfa de Cronbach & N de elementos \\
\hline Ciencias Experimentales & 0,937 & 18 \\
Enseñanza Técnicas & 0,918 & 18 \\
Ciencias de la Salud & 0,949 & 18 \\
Ciencias Sociales y Jurídicas & 0,939 & 18 \\
Humanidades & 0,944 & 18 \\
\hline
\end{tabular}

La correlación de los elementos con el total es elevada, con una correlación media para los 17 de 0,657 siendo el ítem 1 el de correlación más baja $(0,513)$ y el 11 la más alta $(0,766)$. No hay ningún elemento que, en caso de ser eliminado, su ausencia lleve aparejado un incremento del valor del Alfa de Cronbach.

Con objeto de analizar la dimensionalidad del cuestionario, se procedió a efectuar un análisis factorial exploratorio mediante Análisis de Componentes Principales (ACP). Un primer indicador de la estructura dimensional de una prueba es el porcentaje de varianza que explica cada uno de los componentes generados por la solución factorial. Como se observa en la Tabla 7 y en el Gráfico de sedimentación que se presenta a continuación de la misma, el primer componente explica por sí solo el $49,4 \%$ de la varianza total de la prueba. Un segundo indicador son los autovalores de los componentes, y en este caso, sólo los dos primeros son superiores a 1 pero con una gran desproporción entre ambos. A la vista de todo ello, la conclusión es clara: se trata de un instrumento nítidamente unidimensional que por tanto mide básicamente un único rasgo latente.

Tabla 7.- Porcentaje de varianza que explica cada uno de los componentes generados

\begin{tabular}{|c|c|c|c|c|c|c|}
\hline \multirow[b]{2}{*}{ Componente } & \multicolumn{3}{|c|}{ Autovalores iniciales } & \multicolumn{3}{|c|}{$\begin{array}{c}\text { Sumas de las saturaciones al cuadrado de la } \\
\text { extracción }\end{array}$} \\
\hline & Total & $\begin{array}{c}\text { \% de la } \\
\text { varianza }\end{array}$ & $\%$ acumulado & Total & $\begin{array}{c}\text { \% de la } \\
\text { varianza }\end{array}$ & $\%$ acumulado \\
\hline 1 & 8,895 & 49,418 & 49,418 & 8,895 & 49,418 & 49,418 \\
\hline 2 & 1,008 & 5,601 & 55,019 & 1,008 & 5,601 & 55,019 \\
\hline 3 & 0,962 & 5,343 & 60,362 & & & \\
\hline 4 & 0,787 & 4,375 & 64,736 & & & \\
\hline 5 & 0,694 & 3,858 & 68,594 & & & \\
\hline 6 & 0,642 & 3,567 & 72,161 & & & \\
\hline 7 & 0,597 & 3,314 & 75,475 & & & \\
\hline 8 & 0,552 & 3,067 & 78,543 & & & \\
\hline 9 & 0,520 & 2,888 & 81,431 & & & \\
\hline 10 & 0,496 & 2,756 & 84,187 & & & \\
\hline 11 & 0,447 & 2,481 & 86,668 & & & \\
\hline 12 & 0,427 & 2,370 & 89,039 & & & \\
\hline 13 & 0,404 & 2,246 & 91,285 & & & \\
\hline 14 & 0,386 & 2,143 & 93,428 & & & \\
\hline 15 & 0,375 & 2,081 & 95,509 & & & \\
\hline 16 & 0,290 & 1,609 & 97,118 & & & \\
\hline 17 & 0,280 & 1,555 & 98,673 & & & \\
\hline 18 & 0,239 & 1,327 & 100,000 & & & \\
\hline
\end{tabular}




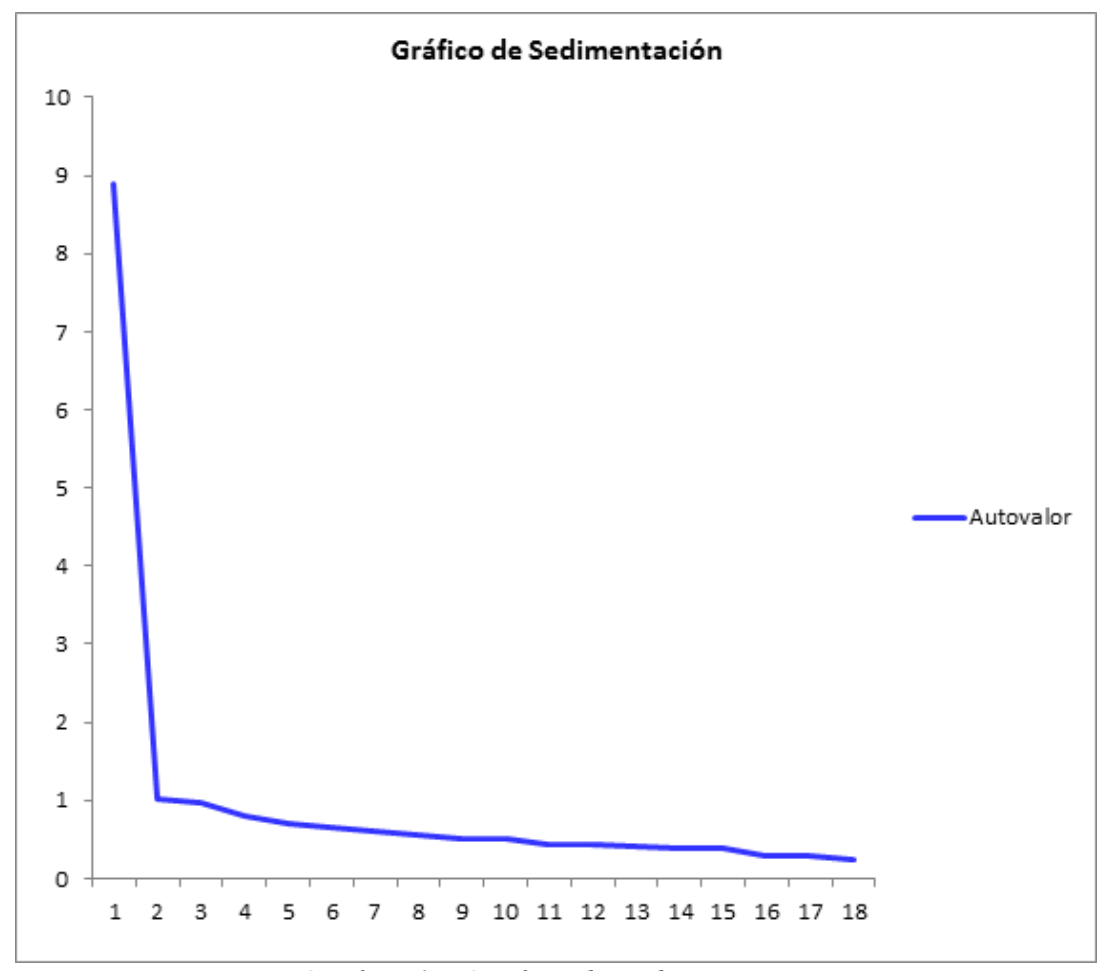

Gráfico 1.- Gráfico de sedimentación

Sin olvidar la clara unidimensionalidad de la prueba, con objeto de definir la estructura factorial de la misma, se ha procedido a efectuar tres tipos de rotaciones: la primera ortogonal y las dos siguientes oblicuas (métodos oblimin y promax). En los tres casos se obtienen definiciones coincidentes, siendo la última la que muestra una estructura de componentes más clara y fácilmente interpretable.

Tabla 8.- Definición de la estructura factorial

\begin{tabular}{ccc}
\hline Ítem & \multicolumn{2}{c}{ Componente } \\
\cline { 2 - 3 } item1 & $\mathbf{1}$ & $\mathbf{2}$ \\
item2 & 0,892 & $-0,319$ \\
item3 & 0,933 & $-0,168$ \\
item4 & 0,439 & 0,318 \\
item5 & 0,650 & 0,062 \\
item6 & 0,614 & 0,141 \\
item7 & 0,793 & 0,013 \\
item8 & 0,395 & 0,351 \\
item9 & $-0,010$ & $\mathbf{0 , 7 4 9}$ \\
item10 & $-0,335$ & $\mathbf{0 , 9 9 6}$ \\
item11 & $-0,051$ & $\mathbf{0 , 7 4 7}$ \\
item12 & 0,452 & 0,414 \\
item13 & 0,558 & 0,186 \\
item14 & 0,627 & 0,139 \\
item15 & 0,215 & 0,567 \\
item16 & 0,390 & 0,363 \\
item17 & 0,567 & 0,270 \\
item18 & 0,413 & 0,402 \\
\end{tabular}


La Tabla 8 muestra la definición de la estructura factorial. En la misma se han resaltado los elementos del cuestionario que tienen alguna contribución reseñable en un hipotético segundo factor. Son los ítems 8, 9 y 10. Aunque, como antes se ha señalado, el cuestionario debe considerarse como claramente unidimensional, un posible segundo componente (que sólo explica el 5,6\% de la varianza) se aglutinaría alrededor de los ítems 8, 9 y 10 en los que parece detectarse un patrón común de respuestas que giran en torno a la promoción del aprendizaje autónomo, el trabajo en equipo y la evaluación de dichas actividades.

Una vez analizada la fiabilidad del instrumento y su estructura dimensional, en este apartado se procede al estudio de la validez del cuestionario. Para ello se analizan las correlaciones de las contestaciones a cada uno de los ítems con las respuestas a los dos ítem criterio incluidos en esta versión del cuestionario (ítem 19: En general, pienso que es un buen profesor o profesora; ítem 20: Si pudiera, me volvería a matricular en otra asignatura impartida por este profesor o profesora). Así mismo se han analizado las correlaciones de cada ítem con la variable promedio resultante del cálculo de la media de los ítems analizados.

Se utilizaron 3 variables como criterio de validez. Los dos primeros son los ítems 19 y 20 y el tercero es el promedio de los 18 ítems iniciales de la prueba. Su funcionamiento y similitud se reflejan en las altas correlaciones que entre ellos se dan: 0,794 y 0,756 entre el promedio y cada uno de los dos ítems criterio; y 0,787 entre estos dos.

En la Tabla 9 y el Gráfico 2 se muestran las correlaciones entre cada ítem y las variablescriterio. En la misma se puede observar lo siguiente:

- Las correlaciones son superiores con la variable criterio promedio.

- Las correlaciones relativamente más bajas con las tres variables-criterio se dan siempre con los ítems 1, 9 y 10. En cualquier caso, su varianza compartida con cada criterio es superior al $10 \%$.

- Las correlaciones con los ítems 19 y 20 son muy similares siendo levemente superiores con el ítem 19.

\begin{tabular}{cccc} 
Tabla 9.- Correlación de cada ítem con los ítems criterio y con el promedio \\
Ítem & $\begin{array}{c}\text { Criterio1 } \\
\text { Item19 }\end{array}$ & $\begin{array}{c}\text { Criterio2 } \\
\text { Item20 }\end{array}$ & Promedio \\
\hline Item1 & 0,416 & 0,382 & 0,570 \\
Item2 & 0,577 & 0,539 & 0,736 \\
Item3 & 0,483 & 0,466 & 0,703 \\
Item4 & 0,491 & 0,449 & 0,674 \\
Item5 & 0,531 & 0,531 & 0,711 \\
Item6 & 0,717 & 0,672 & 0,764 \\
Item7 & 0,569 & 0,540 & 0,690 \\
Item8 & 0,455 & 0,477 & 0,665 \\
Item9 & 0,336 & 0,329 & 0,588 \\
Item10 & 0,389 & 0,386 & 0,631 \\
Item11 & 0,624 & 0,624 & 0,798 \\
Item12 & 0,593 & 0,540 & 0,696 \\
Item13 & 0,674 & 0,635 & 0,722 \\
Item14 & 0,525 & 0,530 & 0,717 \\
Item15 & 0,494 & 0,485 & 0,695 \\
Item16 & 0,622 & 0,589 & 0,776 \\
Item17 & 0,647 & 0,658 & 0,750 \\
Item18 & 0,572 & 0,560 & 0,718
\end{tabular}




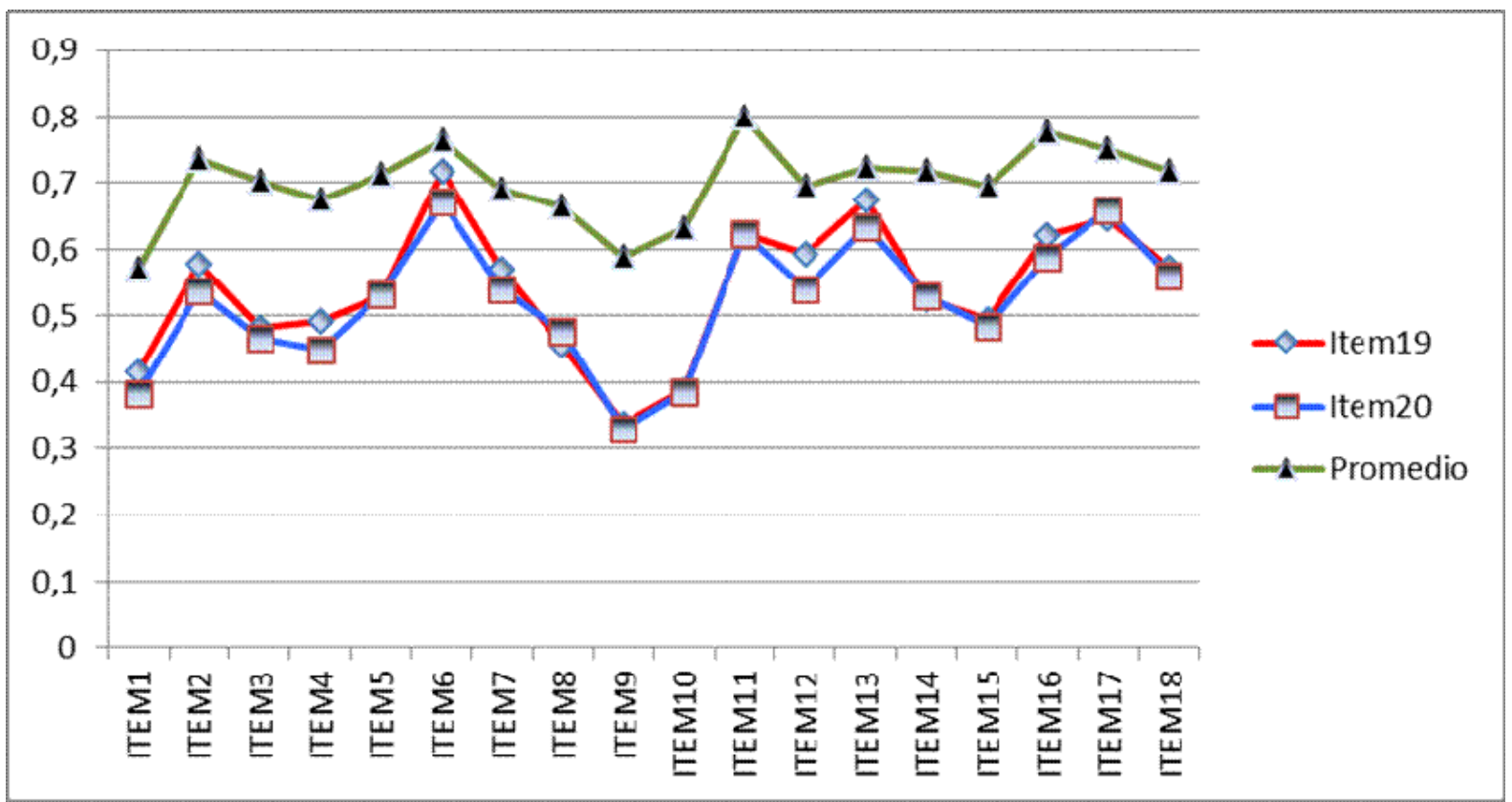

Gráfico 2.- Correlación de cada ítem con los ítems criterio y con el promedio

\section{Discusión}

Tal y como ha podido comprobarse, el cuestionario piloto tiene unas características psicométricas destacadas que indican que el instrumento funciona bien. Posee una alta fiabilidad como consistencia interna similar a la obtenida en otros estudios (Muñoz, Ríos y Abalde, 2002; Bol-Arreba, Sáiz-Manzanares y Pérez-Mateos, 2013). Todos los ítems discriminan suficientemente y en ningún caso la fiabilidad aumenta al eliminar un ítem.

Su estructura es nítidamente unidimensional. Hay que tener en cuenta, que dada la unidimensionalidad del cuestionario por un lado y la alta correlación de todos y cada uno de los ítems con el ítem criterio por otro, la utilización de un único ítem (el criterio) sería suficiente para tener la valoración que hacen los estudiantes del docente. En la línea de lo señalado por Berk (2013), si se optara por la utilización de un único ítem el docente dispondría de la valoración global que hacen sus estudiantes sobre la docencia, pero no tendría a su disposición información acerca de cuáles son los aspectos mejor y peor valorados. Obviamente, si no se dispone de esa información las opciones de mejora por parte del profesor se quedarían muy limitadas si no anuladas. Por ello, conviene que el cuestionario siga manteniendo una serie de ítems clave relacionados con los procesos de enseñanza-aprendizaje deseados en el IKD.

Los resultados han mostrado que el funcionamiento del cuestionario es muy similar tanto en los grados como en los másteres. La conveniencia de tener un único cuestionario para todas las titulaciones también se trató en los grupos de discusión. En este sentido, en general tanto el profesorado como el alumnado se muestran de acuerdo en que lo más conveniente es mantener el mismo cuestionario en todas las titulaciones. Por ello, el cuestionario debe hacer referencia a cuestiones generales que puedan ser aplicables a prácticamente todas las situaciones. De la misma manera, se consideró recomendable que sea el mismo cuestionario el que se utilice en los másteres. Obviamente, un cuestionario de quince o veinte preguntas no puede abarcar la evaluación de todos los aspectos docentes de la asignatura. En este sentido, sería interesante que la universidad creara un Banco de Ítems que pudiera abarcar, si no todos, si la mayoría de los aspectos docentes de las distintas titulaciones de grado y de los másteres. Esta herramienta podría estar disponible para los distintos cargos académicos (e 
incluso para el profesorado) de tal manera que además del cuestionario de opinión del alumnado "oficial", para cada titulación (fuera grado o máster) o asignatura podría crearse el cuestionario ad hoc adecuándose a cada situación.

Aunque en los grupos de discusión se decidió incluir un segundo ítem criterio (el 20) se ha comprobado que la correlación entre los dos ítems criterio (19 y 20) es muy elevada por lo que sería conveniente dejar el cuestionario con un único ítem criterio.

Tal y como puede observarse en el Anexo 1 se presenta el cuadro de dimensiones definitivo obtenido como consecuencia de todo el proceso (tanto cuantitativo como cualitativo) de adaptación realizado. Se han distinguido tres dimensiones tal y como se planteó desde un inicio basándonos en las sugerencias de la ANECA y del modelo IKD. Para cada dimensión se ha explicitado su definición, así como los indicadores que son susceptibles de ser percibidos por el alumnado. Estos indicadores fueron validados en los grupos de discusión tanto con los expertos como con los del profesorado. Por último, en la cuarta columna aparecen los ítems asociados a cada dimensión. En principio, estos serían los ítems propuestos para el cuestionario definitivo.

Un aspecto importante a considerar es la limitación de la información que ofrece un cuestionario de este tipo. Mediante el mismo, se puede conocer la percepción del estudiante con respecto a los aspectos considerados en el cuestionario. Siendo esta percepción importante como medio para la mejora de la docencia, hay que tener en cuenta, tal y como siempre han señalado las distintas instancias de la UPV/EHU, que esta encuesta debe estar integrada en un proceso de evaluación del profesorado más amplio. Es por ello que abogamos por la plena integración de la encuesta de opinión dentro del programa DOCENTIA como un elemento más. Sin embargo, ello no sería suficiente si queremos otorgar a la evaluación del profesorado una función formativa o de mejora de la docencia. El programa de evaluación debe, por lo tanto, estar inmerso en un plan de mejora más amplio que contemple acciones para su consecución. Sería interesante que el profesorado dispusiera de información más cualitativa con respecto a lo que opina el alumnado. Convendría que el cuestionario dispusiera de algunos ítems abiertos en los que el estudiante pudiera mostrar su parecer acerca de la docencia del docente. Esta información podría ser devuelta (feed-back) y de esa manera podría conocer o podría tener una explicación más adecuada de las puntuaciones otorgadas por sus estudiantes. Obviamente esta información podría ser utilizada para su propia mejora. Las dos cuestiones que podrían ser planteadas son las siguientes:

- "Aspectos positivos del docente"

- "Aspectos a mejorar"

En los grupos de discusión mantenidos tanto con los expertos como con el profesorado y el alumnado, se consideró la inclusión de los ítems abiertos como muy positivo.

Lógicamente, en el formato de aplicación actual, la inclusión de ítems abiertos supondría un tiempo de corrección de las respuestas que haría inviable el proceso. Sin embargo, debe plantearse la aplicación del cuestionario por algún medio digital que evite el uso del papel, facilite la corrección y la redacción de los informes. Debería pensarse en aplicar el cuestionario dentro del aula tal y como se realiza hoy en día, pero sustituyendo el papel por algún dispositivo o terminal (ordenador, tableta, teléfono móvil,...). Es decir, la aplicación se realizaría de forma similar a la actual, pero utilizando otro medio. Obviamente, deberá existir un control absoluto para que no pueda ser contestado en más de una ocasión por cada estudiante y no quede rastro de identificación del estudiante que opina. Las ventajas de este procedimiento parecen obvias. Dentro de este marco, cabría la posibilidad de la inclusión de los ítems abiertos mencionados anteriormente. No obstante, convendría que existiera una forma de verificar o asegurar in situ que se han 
tramitado bien las encuestas para que, bien por error o por desinterés del estudiante no haya cuestionarios sin rellenar. En el sistema actual, el docente sabe aproximadamente el número de encuestas entregadas y esto también debería poder conocerse con el sistema on-line.

Otro aspecto controvertido y que ha sido analizado en los distintos grupos de discusión es el relativo a las consecuencias que puede tener para el profesorado la puntuación obtenida en el cuestionario. En este sentido, dependiendo del área de conocimiento se ha criticado la adecuación de algunos de los ítems dado que, como es obvio, no en todas las titulaciones los aspectos recogidos tienen el mismo peso en la docencia. Aspectos tales como el fomento del trabajo en equipo o el desarrollo de la expresión oral y/o escrita por ejemplo, aunque son importantes dentro del modelo IKD de la universidad, no tienen el mismo tratamiento en las distintas titulaciones. Por ello, el profesorado de estas áreas no se mostraba conforme con la inclusión de esos ítems en la suma total de la puntuación del profesorado. No obstante, la política de la UPV/EHU ha sido la de utilizar como puntuación del profesor el último ítem o ítem criterio: "En general, pienso que es un buen profesor o profesora". Dado que se propone el mantenimiento de este ítem, se considera que como puntuación del profesor o profesora debe seguir utilizándose el mismo y no la media de las puntuaciones obtenidas en todos los ítems ya que, como se ha señalado previamente, podría resultar discriminatorio para el profesorado de determinadas áreas. Esto no sucede con el ítem criterio, puesto que independientemente del área considerada se ha comprobado la neutralidad del mismo. El estudiante es capaz de percibir y valorar desde su perspectiva y considerando las circunstancias, las competencias del docente en cuestión.

\section{Referencias}

ANECA (2006). DOCENTIA (Programa de apoyo para la evaluación de la actividad docente del profesorado universitario). Modelo de evaluación. Recuperado de http://www.aneca.es/var/media/215769/docen tia_modelo_070302.pdf

Apodaca, P. \& Grad, H. (2002). Análisis dimensional de las opiniones de los alumnos universitarios sobre sus profesores: comparación entre técnicas paramétricas y no paramétricas. Revista de Investigación Educativa, 20(2), 385-409.

Berk, R. A. (2013). Should Global on Student Rating Scales Bi Used for Summative Decisions?. Journal of Faculty Development, 27(1), 63-67.

Bol-Arreba, A., Sáiz-Manzanares, M.C. \& Pérez-Mateos, M. (2013). Validación de una encuesta sobre la actividad docente en Educación Superior. Aula Abierta, 41(2), 45-54.

Casero Martínez, C. (2008). Propuesta de un cuestionario de evaluación de la calidad docente universitaria consensuado entre alumnos y profesores. Revista de Investigación Educativa, 26(1) 25-44.

Creswell, J.W. \& Plano, V.L. (2011). Designing and conducting Mixed Methods Research. London: SAGE.

De la Fuente, J., Martínez, J.M., Peralta, F.J. \& García, A.B. (2010). Percepción del proceso de enseñanza-aprendizaje y rendimiento académico en diferentes contextos instruccionales de la Educación Superior. Psicothema, 22(4), 806-812.

Gómez-Gallego, J.C., Gómez-Gallego, M., Pérez-Cárceles, M.C., Palazón-Pérez de los Cobos, A. \& Gómez-García, J. (2013). Interacción entre las expectativas académicas del alumno y la evaluación del profesorado. Aula Abierta 2013, 41(2), 35-44.

Lukas, J.F. \& Santiago, K. (2009). Evaluación educativa. Madrid: Alianza.

Marsh, H. W. (1987). Students' evaluations of university teaching: Research findings, methodological issues, and directions for future research. International Journal of Educational Research, 11, 253-288.

Molero, D. \& Ruiz, J. (2005). La evaluación de la docencia universitaria. Dimensiones y variables más relevantes. Revista de Investigación Educativa, 23(1), 57-84. 
Muñiz, J. (2005). La validación de los tests. Metodología de las ciencias del comportamiento, 5, 121-141.

Muñoz Cantero, J.M., Ríos de Deus, M.P. \& Abalde, E. (2002). Evaluación docente vs. Evaluación de la calidad. Revista Electrónica de Investigación y Evaluación Educativa, 8(2). Recuperado de http://www.uv.es/RELIEVE/v8n2/RELIEVE v8n2 4.pdf

Palazón, A. (2011). Evaluación de la docencia y del aprendizaje en el marco del Espacio Europeo de Educación Superior. Tesis doctoral inédita. Universidad Católica San Antonio de Murcia. Recuperado de http://repositorio.ucam.edu/jspui/bitstream/10 952/139/1/Tesis Doctoral pdf.pdf
Ruiz Carrascosa, J. (2005). La evaluación de la docencia en los planes de mejora de la universidad. Educación XXI, 8, 87-102.

Tejedor, F.J. (2003). Un modelo de evaluación del profesorado universitario. Revista de Investigación Educativa, 21(1), 157-182.

Tejedor, F.J. (2009). Evaluación del profesorado universitario: enfoque metodológico y algunas aportaciones de la investigación. Estudios sobre Educación, 16, 79-102.

Worthington, A. (2002). The impact of student perceptions and characteristics on teaching evaluations: a case study in finance education. Assessment and Evaluation in Higher Education, 27(1), 49-64. doi: $\underline{10.1080 / 02602930120105054}$

\section{Anexo 1}

\begin{tabular}{|c|c|c|c|}
\hline Dimensión & Definición & Indicadores & Ítems \\
\hline $\begin{array}{c}\text { Planificación de la } \\
\text { docencia }\end{array}$ & $\begin{array}{l}\text { Esta dimensión se refiere a } \\
\text { todo el proceso previo de } \\
\text { reflexión y diseño de la } \\
\text { docencia de una materia } \\
\text { con objeto de promover un } \\
\text { aprendizaje activo y autó- } \\
\text { nomo. }\end{array}$ & $\begin{array}{l}\text { Resultados de aprendizaje pre- } \\
\text { vistos. Claridad con la que es- } \\
\text { tán planteados los objetivos y } \\
\text { las competencias a desarrollar. } \\
\text { Organización de la enseñanza } \\
\text { (clases prácticas, prácticas ex- } \\
\text { ternas, seminarios, clases teóri- } \\
\text { cas, tutorías, etc.). } \\
\text { Planificación de la enseñanza y } \\
\text { del aprendizaje con relación a } \\
\text { las materias o asignaturas im- } \\
\text { partidas. } \\
\text { Actividades de aprendizaje } \\
\text { previstas. } \\
\text { Criterios y métodos de evalua- } \\
\text { ción. } \\
\text { Materiales y recursos para la } \\
\text { docencia. } \\
\text { Viabilidad de desarrollo efecti- } \\
\text { vo del programa desde la pers- } \\
\text { pectiva del tiempo que debe } \\
\text { invertir el estudiante. }\end{array}$ & $\begin{array}{l}\text { 1. Proporciona una Guía del } \\
\text { Estudiante (programación) útil } \\
\text { para el seguimiento de la asig- } \\
\text { natura. } \\
\text { 2. Planifica de manera ordena- } \\
\text { da la asignatura. } \\
\text { 3. Recomienda (bibliografía, } \\
\text { materiales, TICS, etc.) que } \\
\text { facilitan nuestro aprendizaje. } \\
\text { 4. Comunica claramente al } \\
\text { comienzo de curso los criterios } \\
\text { y procedimientos de evalua- } \\
\text { ción. } \\
5 . \text { Adecúa el nivel de dificultad } \\
\text { de las clases a nuestros cono- } \\
\text { cimientos previos. }\end{array}$ \\
\hline
\end{tabular}




\begin{tabular}{|c|c|c|c|}
\hline Dimensión & Definición & Indicadores & Ítems \\
\hline $\begin{array}{l}\text { Desarrollo de la } \\
\text { docencia e inter- } \\
\text { acción docente }\end{array}$ & $\begin{array}{l}\text { Esta dimensión hace } \\
\text { referencia a la aplicación } \\
\text { de estrategias metodoló- } \\
\text { gicas adecuadas a las } \\
\text { necesidades de las y los } \\
\text { estudiantes de forma que } \\
\text { sean coherentes con los } \\
\text { objetivos y competencias } \\
\text { a desarrollar, y que ten- } \\
\text { gan en cuenta el uso de } \\
\text { los recursos didácticos } \\
\text { adecuados para promover } \\
\text { un aprendizaje activo y } \\
\text { autónomo. } \\
\text { También se refiere a la } \\
\text { motivación, el clima } \\
\text { creado en el proceso de } \\
\text { enseñanza y aprendizaje, } \\
\text { el entusiasmo del docente } \\
\text { y sus relaciones con las y } \\
\text { los estudiantes. }\end{array}$ & 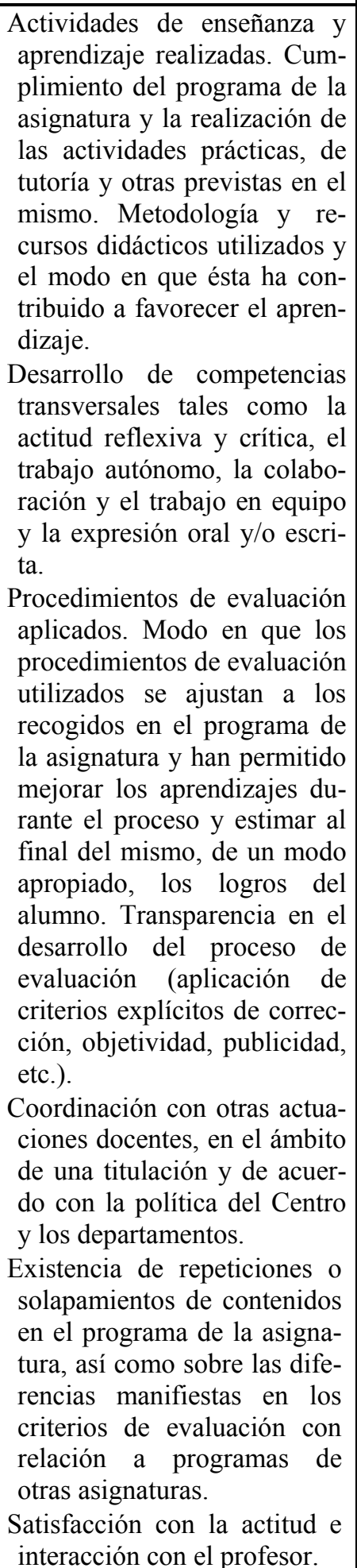 & $\begin{array}{l}\text { 1. Explica de manera clara y } \\
\text { ordenada. } \\
\text { 2. Favorece la actitud reflexi- } \\
\text { va y crítica. } \\
\text { 3. Propone actividades para } \\
\text { favorecer el aprendizaje autó- } \\
\text { nomo (búsqueda de informa- } \\
\text { ción, resolución de casos y } \\
\text { problemas prácticos, investi- } \\
\text { gaciones, etc.). } \\
\text { 4. Estimula la colaboración y } \\
\text { el trabajo en equipo. } \\
\text { 5. Evalúa las actividades que } \\
\text { realizamos y nos informa con } \\
\text { el fin de que podamos mejo- } \\
\text { rar. } \\
\text { 6. Motiva al alumnado para } \\
\text { que se interese por su proceso } \\
\text { de aprendizaje. } \\
\text { 7. Atiende las consultas que le } \\
\text { planteamos. } \\
\text { 8. Transmite entusiasmo im- } \\
\text { partiendo esta asignatura. } \\
\text { 9. Estimula el desarrollo de la } \\
\text { expresión oral y/o escrita. } \\
\text { 10. Se esfuerza para que en- } \\
\text { tendamos la conexión de la } \\
\text { asignatura con el resto de } \\
\text { asignaturas. } \\
\text { 11. Utiliza una metodología y } \\
\text { unos recursos didácticos que } \\
\text { favorecen el aprendizaje. }\end{array}$ \\
\hline
\end{tabular}


Lukas, José Francisco; Santiago, Karlos; Etxeberria, Juan \& Lizasoain, Luis (2014). Adaptación al Es-pacio Europeo de Educación Superior de un cuestionario de opinión del alumnado sobre la docencia de su profesorado.

RELIEVE, 20 (1), art. 3. DOI: 10.7203/relieve.20.1.3812

\begin{tabular}{|l|l|l|l|}
\hline Dimensión & \multicolumn{1}{|c|}{ Definición } & \multicolumn{1}{c|}{ Indicadores } & \multicolumn{1}{c|}{ Ítems } \\
\hline Resultados & $\begin{array}{l}\text { Esta dimensión recoge } \\
\text { aspectos relacionados } \\
\text { con los resultados de }\end{array}$ & $\begin{array}{l}\text { Resultados en términos de } \\
\text { objetivos formativos logra- } \\
\text { dos por los estudiantes. } \\
\text { aprendizaje. }\end{array}$ & $\begin{array}{l}\text { 1. He aprendido mucho cur- } \\
\text { sando esta asignatura. } \\
\text { Grado de desarrollo de las } \\
\text { competencias definidas en la } \\
\text { planificación inicial. } \\
\text { partida con relación a las } \\
\text { competencias previstas. } \\
\text { Satisfacción con la actividad } \\
\text { docente realizada por el pro- } \\
\text { fesor. }\end{array}$ \\
\hline
\end{tabular}

\begin{tabular}{|l|l|l|l|}
\hline \multicolumn{1}{|c|}{ Dimensión } & Definición & Indicadores & \multicolumn{1}{c|}{ Ítems } \\
\hline Ítem Criterio & & & $\begin{array}{l}\text { 1. En general, pienso que es un buen profesor o profesora. } \\
\end{array}$ \\
& & $\begin{array}{l}\text { 2. Si pudiera, me volvería a matricular en otra asignatura im- } \\
\text { partida por este profesor o profesora. }\end{array}$ \\
\hline
\end{tabular}

\begin{tabular}{|c|c|c|c|c|}
\hline \multicolumn{1}{|c|}{ Dimensión } & Definición & Indicadores & \multicolumn{1}{c|}{ Ítems } \\
\hline \multirow{2}{*}{ Ítems abiertos } & & & 1 & Aspectos positivos del docente. \\
& & & 2 & Aspectos a mejorar. \\
\hline
\end{tabular}

\section{ANEXO 2}

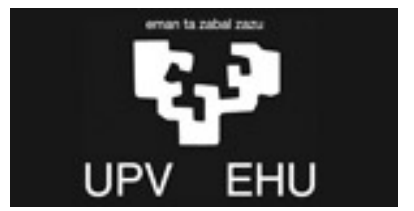

\section{CUESTIONARIO DE OPINIÓN DEL ALUMNADO SOBRE LA DOCENCIA DE SU PROFESORADO}

Para señalar rellena el rectángulo completamente

Si no asistes regularmente a clase, no contestes al cuestionario

Expresa tu valoración según la siguiente escala:

$1=$ Nada de acuerdo

$2=$ Poco de acuerdo

3= Medianamente de acuerdo

$4=$ Bastante de acuerdo

$5=$ Totalmente de acuerdo

$\mathrm{NS} / \mathrm{NC}=$ No sabe/No contesta

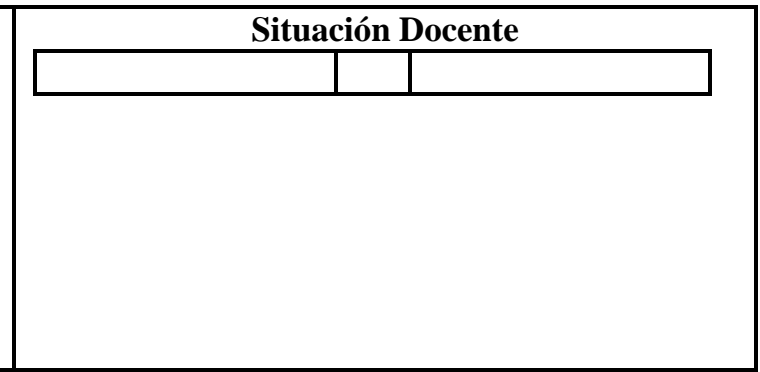

DATOS PARA LA CONTEXTUALIZACIÓN DEL GRUPO DE ALUMNOS Y ALUMNAS::

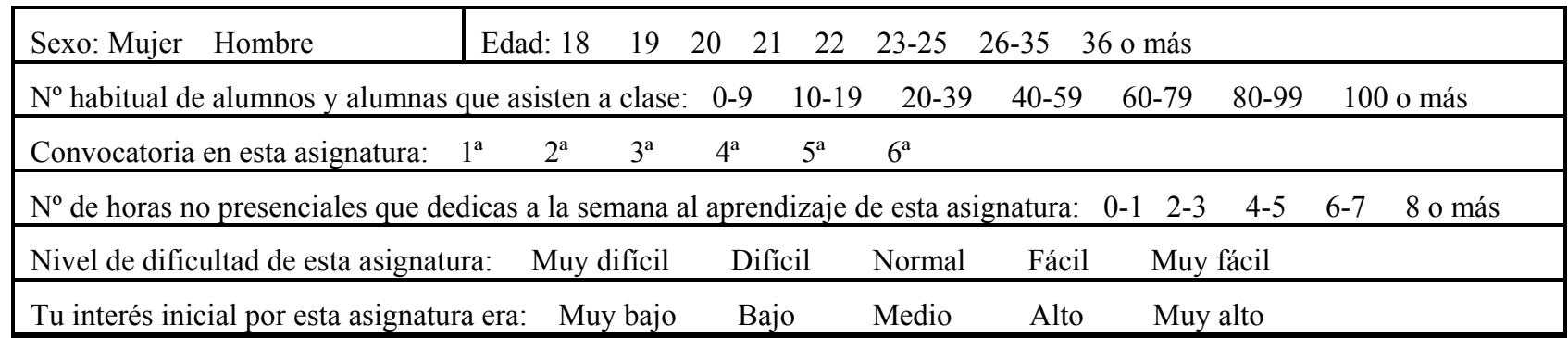


Lukas, José Francisco; Santiago, Karlos; Etxeberria, Juan \& Lizasoain, Luis (2014). Adaptación al Es-pacio Europeo de Educación Superior de un cuestionario de opinión del alumnado sobre la docencia de su profesorado.

RELIEVE, 20 (1), art. 3. DOI: 10.7203/relieve.20.1.3812

\begin{tabular}{|c|c|c|c|c|c|c|}
\hline \multirow{2}{*}{$\begin{array}{l}\text { El profesor o profesora: } \\
\text { 1. Proporciona una Guía del Estudiante (programación) útil para el seguimiento } \\
\text { de la asignatura }\end{array}$} & 1 & 2 & 3 & 4 & 5 & NS/NC \\
\hline & & & & & & \\
\hline 2. Planifica de manera ordenada la asignatura & & & & & & \\
\hline $\begin{array}{l}\text { 3. Recomienda recursos (bibliografía, materiales, TICS, etc.) que facilitan nuestro } \\
\text { aprendizaje }\end{array}$ & & & & & & \\
\hline $\begin{array}{l}\text { 4. Comunica claramente al comienzo de curso los criterios y procedimientos de } \\
\text { evaluación }\end{array}$ & & & & & & \\
\hline 5. Adecúa el nivel de dificultad de las clases a nuestros conocimientos previos & & & & & & \\
\hline 6. Explica de manera clara y ordenada & & & & & & \\
\hline 7. Favorece la actitud reflexiva y crítica & & & & & & \\
\hline $\begin{array}{l}\text { 8. Propone actividades para favorecer el aprendizaje autónomo (búsqueda de } \\
\text { información, resolución de casos y problemas prácticos, investigaciones, etc.) }\end{array}$ & & & & & & \\
\hline 9. Estimula la colaboración y el trabajo en equipo & & & & & & \\
\hline $\begin{array}{l}\text { 10. Evalúa las actividades que realizamos y nos informa con el fin de que poda- } \\
\text { mos mejorar }\end{array}$ & & & & & & \\
\hline 11. Motiva al alumnado para que se interese por su proceso de aprendizaje & & & & & & \\
\hline 12. Atiende las consultas que le planteamos & & & & & & \\
\hline 13. Transmite entusiasmo impartiendo esta asignatura & & & & & & \\
\hline 14. Estimula el desarrollo de la expresión oral y/o escrita & & & & & & \\
\hline $\begin{array}{l}\text { 15. Se esfuerza para que entendamos la conexión de la asignatura con el resto de } \\
\text { asignaturas }\end{array}$ & & & & & & \\
\hline $\begin{array}{l}\text { 16. Utiliza una metodología y unos recursos didácticos que favorecen el aprendi- } \\
\text { zaje. }\end{array}$ & & & & & & \\
\hline 17. He aprendido mucho cursando esta asignatura & & & & & & \\
\hline 18. He mejorado mi nivel de partida con relación a las competencias previstas & & & & & & \\
\hline 19. En general, pienso que es un buen profesor o profesora & & & & & & \\
\hline $\begin{array}{l}\text { 20. Si pudiera, me volvería a matricular en otra asignatura impartida por este } \\
\text { profesor o profesora }\end{array}$ & & & & & & \\
\hline
\end{tabular}

GRACIAS POR TU COLABORACIÓN

\section{NOTA}

Proyecto subvencionado por el Vicerrectorado de Estudios de Calidad e Innovación Docente de la Universidad del País Vasco 


\section{ABOUT THE AUTHORS / SOBRE LOS AUTORES}

Lukas, José Francisco jf.lukas@ehu.es). Doctor en Pedagogía, Licenciado en Filosofía y Ciencias de la Educación (sección Pedagogía) y Diplomado en Magisterio. Profesor Titular de Universidad en Evaluación en Educación en el Departamento de Métodos de Investigación y Diagnóstico en Educación de la Universidad del País Vasco. Especialista en Evaluación en Educación con especial dedicación a temas relacionados con la evaluación de programas y centros educativos y la construcción de instrumentos de medida. Es el autor de contacto para este artículo. Es editor de sección de RELIEVE. Buscar otros artículos de este autor en Google Académico /

\section{Find other articles by this author in Scholar Google Google}

Santiago, Karlos (karlos.santiago@ehu.es). Doctor en Pedagogía, Licenciado en Filosofía y Ciencias de la Educación (sección Pedagogía) y Diplomado en Magisterio. Profesor Titular de Universidad en Métodos de Investigación en Educación en el Departamento de Métodos de Investigación y Diagnóstico en Educación de la Universidad del País Vasco. Es especialista en Métodos de Investigación en Educación con especial dedicación a investigaciones relacionadas con el rendimiento académico y con la evaluación de programas socioeducativos Buscar otros artículos de este autor en Google Académico / Find other articles by this author in Scholar Google Google

Etxeberria, Juan (juanito@ehu.es). Doctor en Ciencias de la Educación y Licenciado en Ciencias Exactas (Especialidad Estadística). Profesor Titular de Estadística en el Departamento de Métodos de Investigación y Diagnóstico en Educación de la Universidad del País Vasco en San Sebastián. Entre los cargos que ostenta, desde el año 1997 es subdirector de los Cursos de Verano que la Universidad del País Vasco organiza en San Sebastián. Buscar otros artículos de este autor en Google Académico / Find other articles by this author in Scholar $\underline{\text { Google }}$

\section{Google}

Lizasoain, Luis (luis.lizasoain@ehu.es). Doctor en Filosofía y Ciencias de la Educación. Profesor Titular de Estadística en el Departamento de Métodos de Investigación y Diagnóstico en Educación de la Universidad del País Vasco en San Sebastián, imparte su docencia tanto en la UPV-EHU como en diversas universidades latinoamericanas. Buscar otros artículos de este autor en Google Académico / Find other articles by this author in Scholar Google Google

\section{ARTICLE RECORD / FICHA DEL ARTÍCULO}

\begin{tabular}{|c|c|}
\hline $\begin{array}{l}\text { Reference / } \\
\text { Referencia }\end{array}$ & 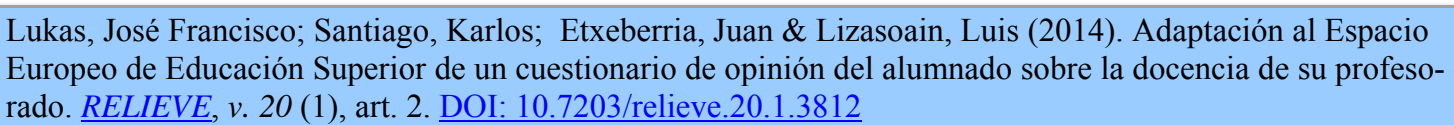 \\
\hline Title / Título & $\begin{array}{l}\text { Adaptación al Espacio Europeo de Educación Superior de un cuestionario de opinión del alumnado sobre la } \\
\text { docencia de su profesorado. [Adapting to the European Higher Education Area a questionnaire on student } \\
\text { opinion about the teaching of lecturers]. }\end{array}$ \\
\hline $\begin{array}{l}\text { Authors / Autores- } \\
\text { tores }\end{array}$ & Lukas, José Francisco; Santiago, Karlos; Etxeberria, Juan \& Lizasoain, Luis \\
\hline Review / Revista & RELIEVE (Revista ELectrónica de Investigación y EValuación Educativa), v. 20 n. 1 \\
\hline ISSN & $1134-4032$ \\
\hline $\begin{array}{l}\text { Publication date / } \\
\text { Fecha de } \\
\text { publicación }\end{array}$ & 2014 (Reception Date: 2014 March 7 ; Approval Date: 2014 June 10. Publication Date: 2014 June 11) \\
\hline
\end{tabular}


Lukas, José Francisco; Santiago, Karlos; Etxeberria, Juan \& Lizasoain, Luis (2014). Adaptación al Es-pacio Europeo de Educación Superior de un cuestionario de opinión del alumnado sobre la docencia de su profesorado.

RELIEVE, 20 (1), art. 3. DOI: 10.7203/relieve.20.1.3812

\begin{tabular}{|c|c|}
\hline $\begin{array}{l}\text { Abstract / } \\
\text { Resumen }\end{array}$ & $\begin{array}{l}\text { The aim of this work is to present the adaptation to the European Higher Education Area of a questionnaire } \\
\text { on the opinions of University of the Basque Country students about their lecturers teaching process. This pro- } \\
\text { cess undertaken at the University used a mixed methodology approach. After reviewing questionnaires from } \\
\text { other universities, taking into account the theoretical framework of the new questionnaire, the transversal } \\
\text { skills adopted by the University and the three dimensions set out by ANECA, a series of indicators and possi- } \\
\text { ble items deemed suitable to be included in the new questionnaire were drawn up. This theoretical framework, } \\
\text { the indicators and the items were all compared and contrasted within various focus groups undertaken with } \\
\text { experts in the topic, with lecturers from the different areas of the University, and with students. Based on this } \\
\text { comparison, the pilot questionnaire, which had } 18 \text { items plus two more criterion-referenced items, was drawn } \\
\text { up. This was applied to a sample of almost one thousand students. The analyses carried out to compare the } \\
\text { efficacy of the items, as well as the reliability and the validity of the test, show that the questionnaire rigor- } \\
\text { ously complies with the standards required by this type of instrument. Finally, in the discussion of the results, } \\
\text { certain controversial aspects, or those relating to improving the evaluation of the university teaching staff, } \\
\text { are presented. Indicated amongst these aspects, is the need to incorporate the questionnaire into a more wid- } \\
\text { er-ranging evaluation plan, such as DOCENTIA, the possibility of creating banks of items, the inclusion of } \\
\text { open items and the exigency of undertaking on-line applications of the questionnaire. }\end{array}$ \\
\hline & $\begin{array}{l}\text { El objetivo de este trabajo es presentar el proceso de adaptación al Espacio Europeo de Educación Superior } \\
\text { del cuestionario de opinión del alumnado sobre la docencia de su profesorado que se ha seguido en la Univer- } \\
\text { sidad del País Vasco. En este proceso se ha adoptado una metodología mixta. Tras efectuar la revisión de los } \\
\text { cuestionarios de otras universidades, teniendo en cuenta el marco teórico del nuevo cuestionario, las compe- } \\
\text { tencias transversales adoptadas por la Universidad y las tres dimensiones que señala la ANECA, se redactaron } \\
\text { una serie de indicadores y posibles ítems susceptibles de conformar el nuevo cuestionario. Este marco teórico, } \\
\text { los indicadores e ítems fueron contrastados en diversos grupos de discusión realizados con expertos en el te- } \\
\text { ma, profesorado de distintas áreas de la propia universidad y alumnado. A partir de este contraste se construyó } \\
\text { el cuestionario piloto. El mismo consta de } 18 \text { ítems más dos ítems criterio. Fue aplicado a una muestra cercana } \\
\text { al millar de alumnos. Los análisis realizados para comprobar la eficacia de los ítems, así como la fiabilidad y } \\
\text { la validez de la prueba señalan que el cuestionario cumple con rigurosidad los estándares exigidos a este tipo } \\
\text { de instrumentos. Por último, en la discusión de los resultados, se presentan algunos aspectos de controversia o } \\
\text { mejora de la evaluación del profesorado universitario. Entre otros, se señala la necesidad de insertar el cues- } \\
\text { tionario en un plan de evaluación más amplio como DOCENTIA, la posibilidad de crear bancos de ítems, la } \\
\text { inclusión de ítems abiertos o la necesidad de realizar aplicaciones on-line del cuestionario. }\end{array}$ \\
\hline $\begin{array}{l}\text { Keywords / } \\
\text { Descriptores }\end{array}$ & $\begin{array}{l}\text { Teacher evaluation; European Higher Education Area; survey validation; reliability; validity; mixed meth- } \\
\text { ods. } \\
\text { Evaluación del profesorado, Espacio Europeo de Educación Superior, validación de encuesta, fiabilidad, vali- } \\
\text { dez, métodos mixtos }\end{array}$ \\
\hline $\begin{array}{l}\text { Institution / } \\
\text { Institución }\end{array}$ & Universidad del País Vasco (España) \\
\hline $\begin{array}{l}\text { Publication site / } \\
\text { Dirección }\end{array}$ & http://www.uv.es/RELIEVE \\
\hline $\begin{array}{l}\text { Language / } \\
\text { Idioma }\end{array}$ & Español \& English version (Title, abstract and keywords in English \& Spanish) \\
\hline
\end{tabular}

\section{RELIEVE}

\section{Revista ELectrónica de Investigación y $\mathbf{E V}$ aluación $\mathbf{E}$ ducativa E-Journal of Educational Research, Assessment and Evaluation}

$$
\text { [ISSN: 1134-4032] }
$$

(C) Copyright, RELIEVE. Reproduction and distribution of this articles it is authorized if the content is no modified and their origin is indicated (RELIEVE Journal, volume, number and electronic address of the document).

(C) Copyright, RELIEVE. Se autoriza la reproducción y distribución de este artículo siempre que no se modifique el contenido y se indique su origen (RELIEVE, volumen, número y dirección electrónica del documento). 